\title{
Improvement of Durability of Hybrid Ceramic Ball Bearings in Liquid Hydrogen at 3 Million DN (120,000 rpm)
}

\author{
Masataka Nosaka ${ }^{1)^{*}}$, Satoshi Takada ${ }^{2)}$, Makoto Yoshida ${ }^{2)}$, \\ Masataka Kikuchi ${ }^{2)}$, Takayuki Sudo ${ }^{2)}$ and Shohei Nakamura ${ }^{3)}$ \\ ${ }^{1)}$ Department of Mechanical Engineering, The University of Tokyo \\ 7-3-1 Hongo, Bunkyo-ku, Tokyo 113-8656, Japan \\ ${ }^{2)}$ Kakuda Space Center, Japan Aerospace Exploration Agency \\ 1 Koganezawa, Kimigaya, Kakuda 981-1525, Japan \\ ${ }^{3)}$ Aerospace Project Team, Industrial Sales Headquarters, NTN corporation \\ 3066 Higashikata, Kuwana 511-8678, Japan \\ *Corresponding author: nosaka@mech.t.u-tokyo.ac.jp
}

( Manuscript received 14 December 2009; accepted 8 February 2010; published 15 February 2010 )

\begin{abstract}
The durability of a hybrid ceramic 25 -mm-bore ball bearing having a single outer land-guided retainer was evaluated and improved in liquid hydrogen at speeds up to $120,000 \mathrm{rpm}\left(120,000 \mathrm{~min}^{-1}\right)(3$ million DN) by comparing the propagation of superficial thermal microcracks on $\mathrm{Si}_{3} \mathrm{~N}_{4}$ balls at various speeds. To select a tough $\mathrm{Si}_{3} \mathrm{~N}_{4}$ ball capable of restraining crack propagation, bearing tests were conducted for three kinds of the $\mathrm{Si}_{3} \mathrm{~N}_{4}$ balls with different jet-cooling systems. The test results showed that the $\mathrm{Si}_{3} \mathrm{~N}_{4}$ ball having high fracture toughness as well as high thermal shock resistance could restrain the propagation of wide-ditch microcracks. With regard to the jet-cooling system, four nozzles were better than two nozzles with increasing jet speed. Furthermore, the jet flow aimed at the retainer was superior to that aimed at the inner race, and its result indicated that microcracks might be generated at the trace in contact with the outer raceway under insufficient cooling conditions with a large centrifugal force. Thus, decreasing the maximum contact stress of the outer race to $2.0 \mathrm{GPa}$ by a limited race curvature of 0.51 could restrain the propagation of superficial microcracks.
\end{abstract}

Keywords: cryogenic tribology, solid-lubrication, ball bearing, hybrid ceramic bearing, $\mathrm{Si}_{3} \mathrm{~N}_{4}$ ball, thermal shock, crush load, thermal cracks, jet cooling, bearing acceleration

\section{Introduction}

Advanced liquid oxygen $\left(\mathrm{LO}_{2}\right) /$ liquid hydrogen $\left(\mathrm{LH}_{2}\right)$ pump-fed rocket engines of light weight and characterized by high performance are required for future space transportation systems to reduce launch costs and to increase efficiency. To realize such engines, it is effective to use high-pressure, high-speed turbopumps. For high-speed turbopumps, which provide cryogenic $\mathrm{LO}_{2} / \mathrm{LH}_{2}$ propellants to a combustor, their rotor speed is typically restricted by the DN (bearing bore, $\mathrm{mm} \times$ rotational speed, $\mathrm{rpm}$ ) limits of the bearings operating under cryogenic conditions to reduce bearing wear. Therefore, a cryogenic high-DN bearing exhibiting high durability is required in the development of such a high-speed turbopump. The all-steel bearings (SUS $440 \mathrm{C}$ ) of the $\mathrm{LH}_{2}$ turbopump used in the LE-7 rocket engine had demonstrated excellent lubricating conditions without severe wear at 2 million DN $(50,000$ $\mathrm{rpm})^{1)}$.

A hybrid ceramic bearing consisting of hard, lightweight $\mathrm{Si}_{3} \mathrm{~N}_{4}$ ceramic balls and steel rings enables higher speed operation because of the lower centrifugal force acting on the ceramic balls and good tribological combinations even under extreme conditions at cryogenic temperatures ${ }^{2}$. Recently, it has been noted that advanced high-performance rocket engines would need turbopumps capable of operating at higher speed (ultra-high speed), i.e. $100,000 \mathrm{rpm}^{3,4)}$. For these ultrahigh-speed turbopumps, hybrid ceramic bearings consisting of $\mathrm{Si}_{3} \mathrm{~N}_{4}$ balls and steel rings have been used. Also, for the improved high-pressure $\mathrm{LO}_{2} / \mathrm{LH}_{2}$ turbopumps of the reusable space shuttle main engine (SSME), hybrid bearings with $\mathrm{Si}_{3} \mathrm{~N}_{4}$ balls were used instead of conventional all-steel bearings in an attempt to reduce serious bearing wear ${ }^{5)}$. 
In previous studies ${ }^{6,7)}$, tribo-characteristics of a new type of hybrid ceramic ball bearing (25-mm bore) having $\mathrm{Si}_{3} \mathrm{~N}_{4}$ balls with a single guided retainer were evaluated in $\mathrm{LH}_{2}, \mathrm{LO}_{2}$, or liquid nitrogen $\left(\mathrm{LN}_{2}\right)$ at speeds to $50,000 \mathrm{rpm}$. It was indicated that the hybrid ceramic bearing was characterized by better self-lubrication and higher load capacity in $\mathrm{LH}_{2}$ than in $\mathrm{LO}_{2}$ or $\mathrm{LN}_{2}$. Furthermore, for this single-guided hybrid ceramic bearing, ultra-high-speed performance was determined in $\mathrm{LH}_{2}$ by increasing speeds up to 120,000 rpm ( 3 million DN) under thrust loads up to $3,140 \mathrm{~N}^{8)}$. The hybrid ceramic bearing exhibited excellent performance at high load, contrasting with the seized all-steel bearing; however, for the tested $\mathrm{Si}_{3} \mathrm{~N}_{4}$ balls, superficial thermal microcracks were formed due to frictional heating under higher thrust loads with poor cooling conditions. It was also found that the critical load capacity (stress) at the inner raceway without bearing damage was about $3.0 \mathrm{GPa}$ with a high spin velocity.

While the RL60 demonstrator engine was developed in the USA with international collaboration (USA, Japan, Russia and Sweden), the $\mathrm{LH}_{2}$ turbopump (rotational speed, 90,000 rpm) was developed by a Japanese company ${ }^{3)}$. In the development of this turbopump in Japan, the results obtained in the present study were applied to the development of ultra-high-speed turbopump bearings $(100,000 \mathrm{rpm}, 2.5$ million DN). Therefore, increasing the durability of the hybrid ceramic bearing by the prevention of the propagation of thermal microcracks was essential to realize the capabilities required for advanced high-speed turbopumps.

On the other hand, for the VINCI engine under development in Europe, high-DN hybrid ceramic bearings were tested in $\mathrm{LH}_{2}$ at a speed of 70,000 rpm (2.8 million $\mathrm{DN}$ ), and $\mathrm{Si}_{3} \mathrm{~N}_{4}$ balls exhibited superficial microcracks ${ }^{4}$. In the course of VINCI $\mathrm{LH}_{2}$ turbopump development, continuous studies on a high-DN bearing operating at DN up to 3.3 million $(120,000 \mathrm{rpm})$ in $\mathrm{LH}_{2}$ have been conducted ${ }^{9)}$. Under severe operating conditions employed for evaluation of safety, C-cracks and pits with microcracks were detected on $\mathrm{Si}_{3} \mathrm{~N}_{4}$ balls at a high Hertzian stress of $3.5 \mathrm{GPa}$; however, catastrophic failure such as a rupture of balls hardy occurred. Furthermore, fatigue caused by spalling on $\mathrm{Si}_{3} \mathrm{~N}_{4}$ balls was evaluated by a long-time bearing test at heavy loading in $\mathrm{LN}_{2}{ }^{10)}$.

In contrast, for the RD0146 engine which was developed for upper stages in Russia, it was published that a rotor speed of the main $\mathrm{LH}_{2}$ turbopump was $123,000 \mathrm{rpm}$ (3.08 million DN) to allow max efficiency of pumps and turbine and to reduce the turbopump weight; however, detail of its bearing was unknown ${ }^{11)}$.

As mentioned above, research and development of a high-DN bearing operating at a level of 3 million DN $(120,000 \mathrm{rpm})$ in $\mathrm{LH}_{2}$ have been actively underway. The purpose of the present study was to evaluate and increase the durability of the hybrid ceramic 25mm-bore bearing in comparison with the propagation of thermal microcracks on the $\mathrm{Si}_{3} \mathrm{~N}_{4}$ balls. This paper was based on a previous study ${ }^{12)}$ for a hybrid ceramic ball bearings tested in $\mathrm{LH}_{2}$ at speeds up to $120,000 \mathrm{rpm} \mathrm{(3}$ million DN). To select a tough ball capable of withstanding microcracks, bearings employing three kinds of the candidate $\mathrm{Si}_{3} \mathrm{~N}_{4}$ balls were tested in $\mathrm{LH}_{2}$ at speeds up to $120,000 \mathrm{rpm}$. The bearing tests were conducted under thrust loads up to $1,960 \mathrm{~N}$ and various jet-cooling systems under poor cooling conditions.

Attention was focused on the thermal-shock damage of $\mathrm{Si}_{3} \mathrm{~N}_{4}$ balls, on the improvement of jet-cooling ability and on the reduction of bearing-stress conditions, restraining the propagation of thermal microcracks. The critical load capacity of transferred lubricant film to prevent the propagation of microcracks, namely, that which could withstand high stress due to centrifugal force at the outer raceway, was also estimated.

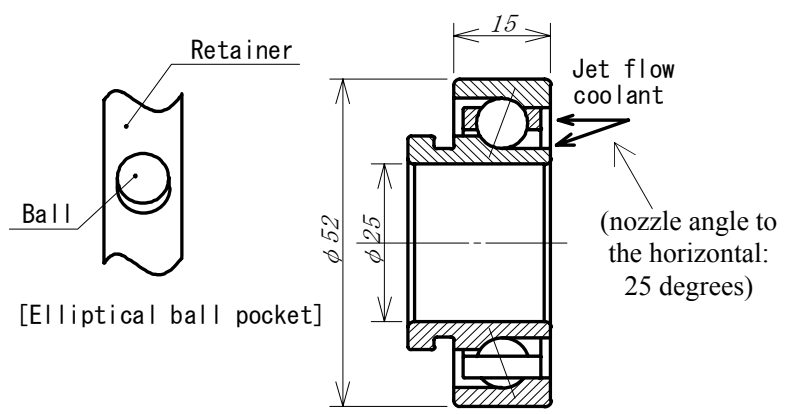

Fig. 1 Configuration of single-guided bearing

\section{Test apparatus and procedure}

\subsection{Test bearings}

Figure 1 shows the configuration of an angular contact, hybrid ceramic $25-\mathrm{mm}$-bore ball bearing ${ }^{1)}$. The test bearings had SUS $440 \mathrm{C}$ rings, as well as $\mathrm{Si}_{3} \mathrm{~N}_{4}$ balls, and a single outer land-guided retainer with ellipticalshaped ball pockets. The circumferential clearance of elliptical pockets was twice as large as that of the conventional circular pockets so as to reduce ball-topocket interaction induced by the ball-speed-variation (BSV). Its axial clearance was considerably narrow so as to stabilize wobbling of the single-guided retainer at high speed ${ }^{1)}$.

This single-guided bearing was able to reduce the bearing torque to about one-half of that of the conventional double-guided bearing, and more adequate cooling at the outer raceway was obtained, so that high self-lubricating performance and high durability were achieved $^{6)}$. The bearings were self-lubricated with a retainer consisting of glass cloth-reinforced PTFE (polytetrafluoroethylene) (glass cloth, 45 mass $\%$ and PTFE, 55 mass\%). To improve self-lubricating performance, abrasive glass-cloth exposed on the retainer surface was chemically etched with hydrofluoric acid 
(HF), and the retainer surface was smoothed. Furthermore, the hybrid ceramic bearing exhibited better load capacity than that of the all-steel bearing due to the tribo-chemical formation of a thicker transfer film consisting of $\mathrm{CaF}_{2} / \mathrm{FeF}_{2}$ in $\mathrm{LH}_{2}{ }^{7}$.

Table 1 contains a summary of the main design parameters of the test bearings. The test results indicated that thermal microcracks on the ball might be generated at the track in contact with the outer raceway under high centrifugal loading. Therefore, to decrease the outerrace contact stress on the modified bearing, the outerrace curvature was decreased to a limited value of 0.51 . For the modified bearing $\mathrm{A}$, the outerland clearance was conventional $(0.2-0.3 \mathrm{~mm})$. In contrast, to clarify how the poor cooling conditions affected the propagation of microcracks, a retainer with a narrow outer-land clearance of 0.1-0.2 mm was employed for the modified bearing $\mathrm{B}$ and decreased the cooling flow rate through the outerland clearance.

Rotor growth due to centrifugal force results in a reduction of the radial clearance within the bearing, resulting in a decrease in the initial contact angle and an increase in the contact stress. For example, at a speed of $120,000 \mathrm{rpm}$, the initial radial clearance of $77 \mu \mathrm{m}$ was consequently decreased to $43 \mu \mathrm{m}$ due to high centrifugal force.

Table 1 Main design parameters of test bearings

\begin{tabular}{|c|c|c|}
\hline DESIGN PARAMETERS & Conventional Bearing & Modified Bearing \\
\hline [BEARING] & \\
\hline Dimensions (mm) & \multicolumn{2}{|c|}{$\Phi 25 \times \Phi 52 \times 15$} \\
\hline Pitch diameter (mm) & \multicolumn{2}{|c|}{38.5} \\
\hline Ball diameter (mm) & \multicolumn{2}{|c|}{7.938} \\
\hline Initial contact angle (deg.) & \multicolumn{2}{|c|}{20} \\
\hline Race curvature (inner/outer) & $0.56 / 0.52$ & $0.56 / 0.51$ \\
\hline Radial clearance $(\mu \mathbf{m})$ & 77 & 67 \\
\hline Number of balls & \multicolumn{2}{|c|}{10} \\
\hline [RETAINER] & \multirow{3}{*}{\multicolumn{2}{|c|}{ Elliptical pocket }} \\
\hline Ball pocket & & \\
\hline Ball pocket clearance: & & \\
\hline Circumferential/axial (mm) & \multicolumn{2}{|c|}{$1.2-1.3 / 0.1-0.2$} \\
\hline Outer land clearance (mm) & $0.2-0.3$ & $0.2-0.3(\mathrm{~A}) / 0.1-0.2(\mathrm{~B})$ \\
\hline Retainer guidance & \multicolumn{2}{|c|}{ Single outer land-guided } \\
\hline
\end{tabular}

\subsection{Candidate $\mathrm{Si}_{3} \mathrm{~N}_{4}$ balls}

To select a tough $\mathrm{Si}_{3} \mathrm{~N}_{4}$ ball capable of restraining crack propagation, three kinds of the candidate $\mathrm{Si}_{3} \mathrm{~N}_{4}$ balls were tested. Table 2 shows a summary of the material parameters of the tested $\mathrm{Si}_{3} \mathrm{~N}_{4}$ balls obtained from the catalog data. The $\mathrm{Si}_{3} \mathrm{~N}_{4}$ balls were hot-pressed material containing $\mathrm{Al}_{2} \mathrm{O}_{3}-\mathrm{Y}_{2} \mathrm{O}_{3}$ as a densification aid. Both balls $\mathrm{A}$ and $\mathrm{C}$ were manufactured by the same company. For characteristic properties of these $\mathrm{Si}_{3} \mathrm{~N}_{4}$ balls, in particular, the ball $\mathrm{A}$ had a higher thermal conductivity than those of the balls $\mathrm{B}$ and $\mathrm{C}$ to increase the thermal stress resistance; however, mechanical strength relatively reduced. In contrast, to reduce mechanical damage during operation, the ball $\mathrm{B}$ had a higher fracture-toughness and the ball $\mathrm{C}$ had a higher bending strength, respectively.

Table 2 Material parameters of $\mathrm{Si}_{3} \mathrm{~N}_{4}$ balls from catalog

\begin{tabular}{|l|c|c|c|}
\hline \multicolumn{1}{|c|}{ BALLS } & A & B & C \\
\hline Density $\times 10^{3}\left(\mathrm{~kg} / \mathrm{m}^{3}\right)$ & 3.20 & 3.25 & 3.20 \\
Three-point bending strength $(\mathrm{MPa})$ & 1000 & 1100 & 1200 \\
Poisson's ratio & 0.27 & 0.28 & 0.27 \\
Young's modulus $(\mathrm{GPa})_{\text {Fracture toughness }\left(\mathrm{MPa} \mathrm{m}^{1 / 2}\right)}^{320}$ & 310 & 312 \\
${\text { Thermal expansion ratio } \times 10^{-6}(/ \mathrm{K})^{\text {a) }}}^{\text {a) }}$ & 5.5 & 6.3 & 5.2 \\
${\text { Thermal conductivity }(\mathrm{W} / \mathrm{m} \mathrm{K})^{\text {b) }}}$ & 31 & 3.4 & 2.9 \\
\hline
\end{tabular}

Table 3 Calculated thermal-shock parameters

\begin{tabular}{|c|c|c|c|}
\hline BALLS & $\mathrm{R}(\mathrm{K})$ & $\mathrm{R}(\mathrm{W} / \mathrm{m})$ & $\mathrm{R}^{\prime \prime}(/ \mathrm{MPa})$ \\
\hline $\mathrm{A}$ & 815 & $2.53 \times 10^{4}$ & 0.438 \\
$\mathrm{~B}$ & 751 & $1.50 \times 10^{4}$ & 0.356 \\
$\mathrm{C}$ & 968 & $2.52 \times 10^{4}$ & 0.297 \\
\hline
\end{tabular}

To evaluate thermal shock resistance of candidate balls, traditional thermal-shock parameters $\left.{ }^{13}\right)\left(R, R^{\prime}\right.$ and $R$ "') were calculated using above catalog data. The calculated results are shown in Table 3. Here, the thermal stress resistance parameters $\left(R\right.$ and $\left.R^{\prime}\right)$ and the thermal shock damage resistance parameter $(R$ "') were as follows ${ }^{13)}$.

$R=\sigma_{f}(1-v) / E \alpha$

$R^{\prime}=\sigma_{f}(1-v) k / E \alpha$

$R^{\prime \prime}=E / \sigma_{f}^{2}(1-v)$

where $\sigma_{f}$ is the bending strength, $v$ is Poisson's ratio, $E$ is Young's modulus, $\alpha$ is the thermal expansion ratio, and $k$ is the thermal conductivity.

It was noted that the thermal stress resistance parameter was appropriate for resistance to initiation of fracture by thermal stress. On the other hand, the thermal shock damage resistance parameter expressed the degree of possibility for further damage caused by thermal shock, and gave information about the minimum in the elastic energy at fracture available for crack propagation. It was suggested that the parameter $R$ "' was useful for comparing the thermal shock resistance of brittle ceramics. To increase the thermal shock damage resistance, there must be lower values of strength and high values of Young's modulus of elasticity and Poisson's ratio ${ }^{13)}$.

The calculated results shown that the ball A indicated the highest value of $R$ "' ' and was followed by that of the ball $\mathrm{B}$. The ball $\mathrm{C}$ resulted in the lowest value of $R$ "' due to high mechanical strength. This tendency was found to be opposite to those of the calculated 
thermal stress resistance parameters ( $R$ and $\left.R^{\prime}\right)$.

Before the bearing tests, to evaluate toughness for three kinds of the $\mathrm{Si}_{3} \mathrm{~N}_{4}$ balls, thermal-shock and crushload tests were conducted. For the thermal-shock test, thermal shock was given by dropping a ball (ball diameter, $7.938 \mathrm{~mm}$ ) heated up to $1,373 \mathrm{~K}$ into water (291 K). The crush load required to rupture two balls (ball diameter, $7.938 \mathrm{~mm}$ ) was detected by loading them against each other at room temperature. From the crush load, the maximum contact-stress was calculated according to conventional Hertzian-contact theory.

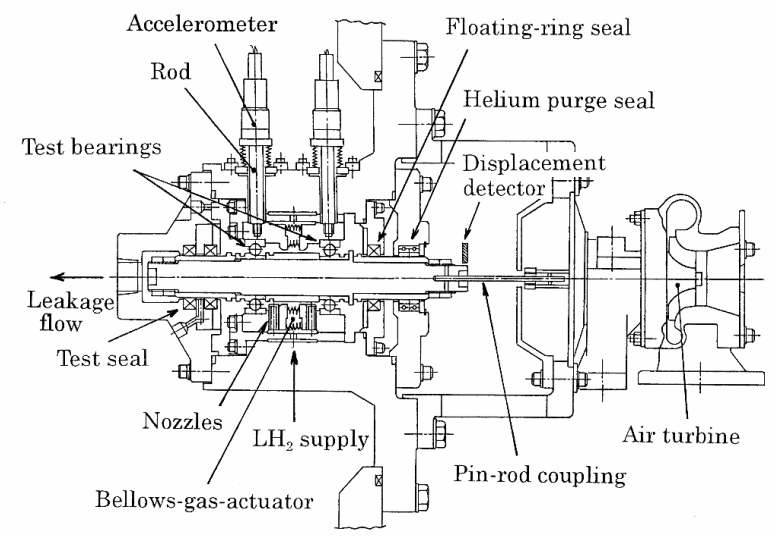

Fig. 2 Configuration of bearing tester

\subsection{Bearing tests}

Figure 2 shows the configuration of the employed bearing tester ${ }^{8)}$. The rotating shaft was supported by two test bearings in the bearing cavity and was driven by an air turbine. Each test bearing was effectively cooled by the jet flow through four or two nozzles facing the center of the retainer's flat side or the inner raceway. A bellows-type gas actuator pressurized by helium gas imposed a thrust load on the test bearings. The pressurized bearing-cavity was sealed with a floating-ring seal having one seal ring for prevention of seal-ring seizure ${ }^{9)}$. Furthermore, the $\mathrm{LH}_{2}$ leakage from the floating-ring seal located at the turbine side was sealed with a segmented circumferential seal which was paired and purged with helium gas.

The test bearings were evaluated in $\mathrm{LH}_{2}$ at speeds up to $120,000 \mathrm{rpm}$ under step-raised thrust-loads of $980 \mathrm{~N}$, $1,470 \mathrm{~N}$ and 1,960 N. The jet-cooling ability was also evaluated by changing the placement and number of nozzles with various inner-diameters of $0.7-1.1 \mathrm{~mm}$, as shown in Fig. 3. Each test bearing was effectively cooled by a jet flow through two or four nozzles aimed at the retainer or the inner raceway, as shown in Fig. 1. For nozzle systems A, B and D, the jet flow through nozzles was aimed at the center of the retainer's flat side. To increase of the cooling ability at the inner race heated by high ball-spinning, nozzle system $\mathrm{C}$ employed two nozzles aimed at the contact track of the inner race (nozzle angle to the horizontal, 25 degrees) in company with two nozzles aimed at the retainer.

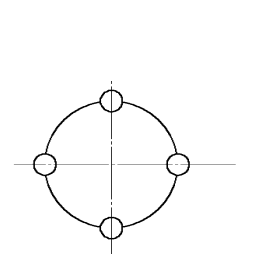

(a) Nozzle systems A and B

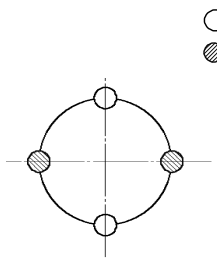

(b) Nozzle system C
Retainer cooling nozzle

Inner-race cooling nozzle

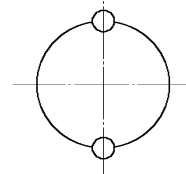

(c) Nozzle system D
- Bearing cooling flow rate, 0.32 liters/s

- Nozzle diameter, $0.7 \mathrm{~mm}$ (A); $0.8 \mathrm{~mm}$ (B); $0.8 \mathrm{~mm}$ (C); $1.1 \mathrm{~mm}$ (D)

- Jet speed, $208 \mathrm{~m} / \mathrm{s}(\mathrm{A}) ; 159 \mathrm{~m} / \mathrm{s}$ (B and C); $168 \mathrm{~m} / \mathrm{s}$ (D)

Fig. 3 Jet-cooling nozzle systems

The cooling flow rate for each bearing was restrained to about 0.32 liters/s, and the jet speed through the nozzles was compared. Thus, the bearings were cooled under a minimum flow rate, resulting in an increase of microcrack propagation in poor cooling conditions. Under a cooling rate of 0.32 liters $/ \mathrm{s}$, the jet speed was $208 \mathrm{~m} / \mathrm{s}$ for the nozzle system A and in a range of $159-168 \mathrm{~m} / \mathrm{s}$ for nozzle systems B, C and D, respectively. To prevent the cooling flow from vaporizing, the bearing-cavity pressure was controlled to maintain it above the critical pressure $(1.29 \mathrm{MPa})$ of $\mathrm{LH}_{2}$.

The bearing temperature was measured at the center of the outer-race surface. In an attempt to directly detect the bearing damage that occurred during operation, the bearing-cartridge-acceleration (BCA) monitoring system was applied ${ }^{14)}$. An accelerometer was mounted at the outside end of the rod, the opposite end of which was screwed onto the bearing cartridge. Analysis of the detected BCA, that is, $G_{r m s}$ (root-mean-square value) and $G_{p k}$ (peak value), was conducted using a conventional acceleration monitor. The BCA as recorded on the chart shows that, for a normal condition of a bearing without damage, $G_{r m s}$ and $G_{p k}$ overlap due to scaling in which five times $G_{r m s}$ equals $G_{p k}$.

\section{Results and discussion}

\subsection{Evaluation of $\mathrm{Si}_{3} \mathrm{~N}_{4}$ balls}

\subsubsection{Thermal-shock and crush-load tests}

Figure 4 shows appearances of the $\mathrm{Si}_{3} \mathrm{~N}_{4}$ balls after the thermal-shock test in water. Furthermore, the crush load and the maximum contact stress determined by the crush-load test are shown in Table 4 . The ball A, which had the lowest strength (crush load) as well as the highest value of $R$ ", , visually indicated no sign of thermal cracks on the ball surface. In addition, the material of ball A had a higher thermal conductivity compared with those of balls B and C. For the ball B, slight cracks appeared at the ball surface, but ball rupture into pieces was prevented. In contrast, the ball C, 
which had the highest strength and the lowest value of $R$ "', was completely ruptured in several pieces due to thermal shock damage.

It was found that the $\mathrm{Si}_{3} \mathrm{~N}_{4}$ ball indicating higher thermal shock resistance owed relatively lower mechanical strength as well as higher thermal conductivity. The tendency of thermal shock damage for the candidate balls was found to be depended on the thermal shock damage resistance parameter $(R$ "') as compared with on the thermal stress resistance parameters ( $R$ and $R$ ”).

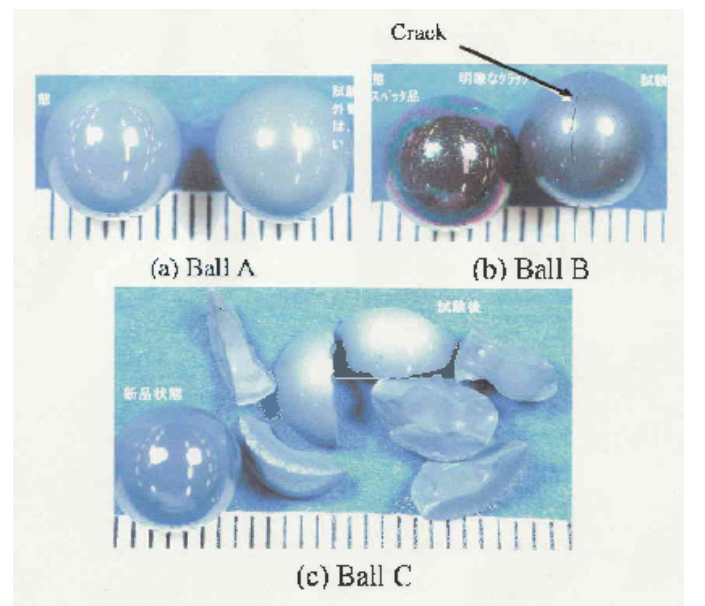

Fig. 4 Appearances of $\mathrm{Si}_{3} \mathrm{~N}_{4}$ balls after thermal-shock test (untested ball on the left)

Table 4 Crush load and maximum contact-stress of $\mathrm{Si}_{3} \mathrm{~N}_{4}$ balls

\begin{tabular}{|l|c|c|c|}
\hline \multicolumn{1}{|c|}{ BALLS } & A & B & C \\
\hline Crush load (kN) & 10.8 & 12.3 & 19.6 \\
Maximum contact stress (GPa) & 25.1 & 25.7 & 30.1 \\
\hline
\end{tabular}

\subsubsection{Bearing tests}

\section{Comparison of ball $A$ vs. ball $B$}

To evaluate the thermal shock resistance affected on the propagation of microcracks, a comparison of the bearings using ball A (which indicated no sign of cracks) and ball B (which showed slight cracks) was conducted. A bearing test was conducted at a lower speed of 100,000 rpm under a light thrust-load of $980 \mathrm{~N}$. In the evaluation of the durability of the bearing with ball $\mathrm{B}$, a total test time of 60 minutes was achieved. In contrast, testing of the bearing with ball A was stopped after 30 minutes due to the formation of wide-ditch cracks. In these tests, the four-nozzle system C, including two nozzles aimed at the inner-race side, was used. As discussed later, nozzle system $\mathrm{C}$ resulted in a reduction of cooling ability at the outer-race side, so that microcracks were apt to be propagated.

Both bearings were successfully operated during tests with a stable change of the bearing temperature, that is, 24.0-24.5 $\mathrm{K}$ for ball $\mathrm{A}$ and $23.5-23.7 \mathrm{~K}$ for ball $\mathrm{B}$, respectively. No remarkable difference of the bearing temperature was detected. With regarding to the BCA of the bearing with ball B detected for 60 minutes, $G_{r m s}(10$ $\mathrm{g})$ and $G_{p k}(50 \mathrm{~g})$ continued to overlap. The change of $\mathrm{BCA}$ resulted in a normal condition without severe damage; however, superficial microcracks were propagated, as shown in Fig. 5 (b). In contrast, for the bearing with ball A, the ratio $G_{r m s}(22 \mathrm{~g}): G_{\text {peak }}(90 \mathrm{~g})$ was detected for 30 minutes, which was slightly higher than expected. Therefore, surface damage with resultant roughness on the balls should have relatively increased; however, hardly any apparent difference was seen for both bearing testing.

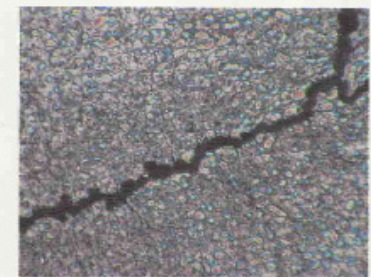

(a) Ball $\Lambda$

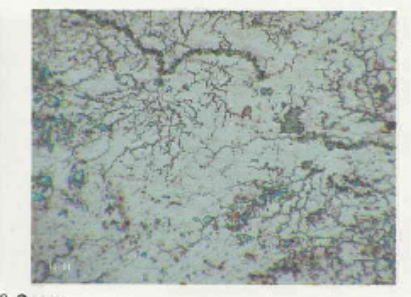

(b) Ball $\mathrm{B}$
Fig. 5 Surface features of balls A and B cooled with nozzle system $\mathrm{C}$

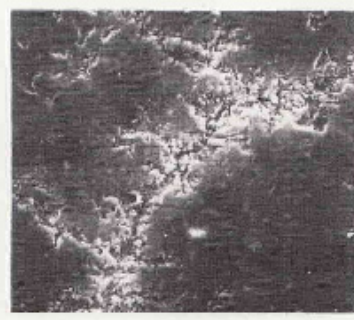

(a) Hair crack

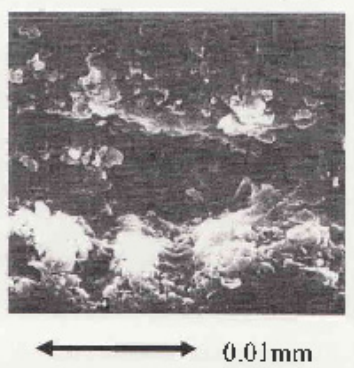

(b) Wide-ditch crack
Fig. 6 Hair cracks and wide-ditch cracks formed on ball A (observed with SEM)

Figure 5 shows typical surface features of the balls A and $\mathrm{B}$ after the test for 30 minutes to check the bearing wear. For the ball B having a high fracture toughness, microcracks appeared to be relatively light as compared with those of ball A. Although the ball A indicated the highest thermal-shock resistance by the thermal-shock test, unexpected wide-ditch cracks with minute webs were mainly observed on all the balls. Microcracks visually extended in a mesh-like pattern and networks of hair cracks propagated along wide- ditch cracks.

The typical features of wide-ditch cracks and hair cracks observed on ball A are shown in Fig. 6. From detailed observation with a scanning electron microscope (SEM), a marked feature of wide-ditch 
cracks was that their depths were very shallow and superficial fractures with flaking occurred along hair cracks. Thus, such wide-ditch cracks seemed to be formed by removal of fragments fractured due to contact stress repeated by the rolling balls, as shown in Fig. 7. Thus, wide-ditch cracks were apt to be formed due to lower mechanical strength and fracture toughness. For several balls tested for 30 minutes, the depth of the superficial microcracks was 4.2-8.4 $\mu \mathrm{m}$ for ball $\mathrm{A}$ and 2.6-6.1 $\mu \mathrm{m}$ for ball $\mathrm{B}$, respectively. Thus, these test results showed that the bearing with ball A exhibited poor resistance to the formation of wide-ditch cracks as compared with that of the bearing with ball B.

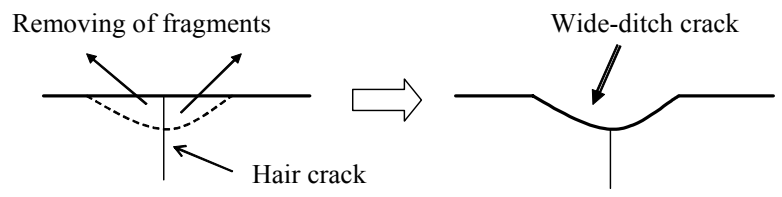

Fig. 7 Process model of wide-ditch crack formation

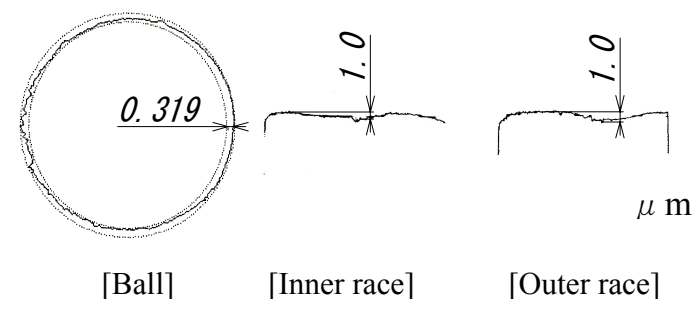

Fig. 8 Wear of ball B and inner-outer raceways tested for 60 minutes

For the bearing with ball $\mathrm{B}$ tested additionally for 60 minutes, the deviation from the spherical form of a ball and the surface profiles of inner-outer raceways of the bearing are shown in Fig. 8. For all balls, the deviation from the spherical form was in a range of 0.14-0.32 $\mu \mathrm{m}$ and the wear depth of the inner-outer raceways was 0.8-1.1 $\mu \mathrm{m}$. In spite of poor cooling conditions using nozzle system $\mathrm{C}$, the bearing components exhibited excellent wear conditions with smooth surfaces. Consequently, it was indicated that microcracks formed during a test time of 30 minutes were scarcely propagated when total time was extended to 60 minutes. Comparison of ball $B$ vs. ball $C$

The bearings having ball $B$ (which showed slight cracks) and ball $\mathrm{C}$ (which was ruptured due to thermal shock) were tested for a total time of 30 minutes under severe conditions; rotational speed was increased to $120,000 \mathrm{rpm}$ under thrust-loads step-raised to $1,960 \mathrm{~N}$ with the use of the four-nozzle system A. It was noticed that increasing the jet speed enabled improvement of the cooling ability within the bearing ${ }^{8)}$. In a previous study ${ }^{8)}$, to gain a more effective jet-cooling flow through the outerland clearance, the jet speed through the two nozzles was found to require more than $197 \mathrm{~m} / \mathrm{s}$ with a cooling flow rate of 0.31 liters/s. Therefore, for the four-nozzle system A, the jet speed was increased to 208 $\mathrm{m} / \mathrm{s}$ with a cooling flow rate of 0.32 liters $/ \mathrm{s}$. It was noted that a jet speed of $208 \mathrm{~m} / \mathrm{s}$ was twice the sliding speed of $108 \mathrm{~m} / \mathrm{s}$ at the outerland of the retainer.

Figure 9 shows the change of the bearing temperature for the bearings having balls $\mathrm{B}$ and $\mathrm{C}$ at a thrust load of $1,960 \mathrm{~N}$ during a total time from 21 minutes to 30 minutes. For the bearing with ball $\mathrm{C}$, the bearing temperature was higher and erratically changed with large variation of $25-28 \mathrm{~K}$. To the contrary, for the bearing with ball $\mathrm{B}$, a stable change at the bearing temperature of $24 \mathrm{~K}$ was exhibited and bearing performance showed better behavior rather than the bearing with ball C.

For both bearings tested for 30 minutes, the wear depth of the inner-outer raceways was at the same level of $0.6-0.8 \mu \mathrm{m}$, although the deviation from the spherical form was $0.14-0.15 \mu \mathrm{m}$ for ball B less severe and 0.25 $0.30 \mu \mathrm{m}$ for ball C. Surface observation also indicated that the few microcracks which formed on ball $\mathrm{B}$ seemed to be relatively superior to ball $\mathrm{C}$.

Thus, above test results showed that the bearing using ball $\mathrm{B}$ exhibited the best bearing performance with prevention of superficial microcrack (hair crack and wide-ditch crack) propagation. It was indicated that the ball B, which had high fracture toughness as well as high thermal-shock resistance, could relatively restrain the propagation of microcracks, resulting in a decrease of ball wear.

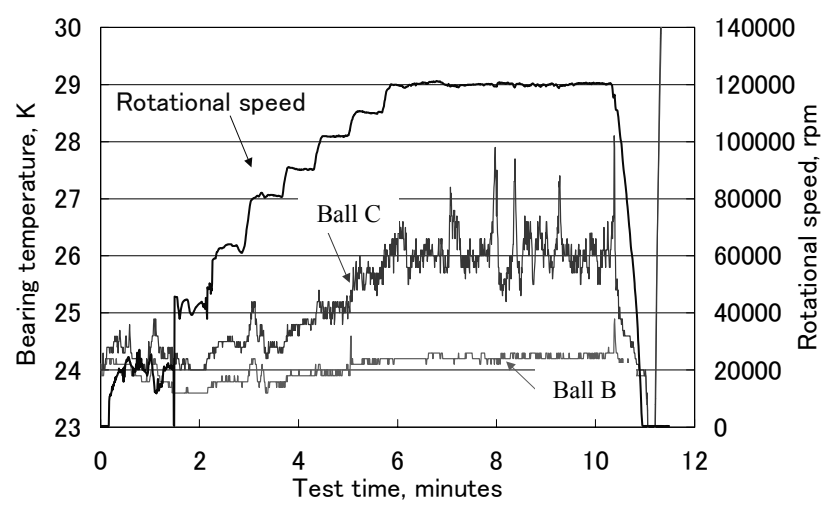

Fig. 9 Change of bearing temperature for bearings used balls $\mathrm{B}$ and $\mathrm{C}$ cooled with nozzle system A

\subsection{Evaluation of jet-cooling systems}

\subsubsection{Jet cooling at retainer and inner race}

A comparison of the cooling ability was conducted for the four-nozzle systems B and C. All nozzles of system B were aimed at the retainer side. For system C, to cool the inner raceway heated by severe ball spinning, two nozzles were aimed at the inner race. To clarify the 
effect of jet-flow direction on cooling ability for both nozzle systems, the jet speed was restrained to be 159 $\mathrm{m} / \mathrm{s}$, so that the jet-cooling effect was lower as compared with that of $208 \mathrm{~m} / \mathrm{s}$ (as shown in Fig. 9). The bearings with tough ball $\mathrm{B}$ were tested for a total time of 30 minutes at speeds to $120,000 \mathrm{rpm}$ under thrust loads step-raised to $1,960 \mathrm{~N}$. Figure 10 shows the change of the bearing temperature in the case of a light thrust load of 980 N. For nozzle system C, in spite of a comparatively light load, the outer-race temperature changed fairly irregularly, and thus poor cooling within the bearing was shown. In contrast, for nozzle system B, no abnormal rise of the bearing temperature was detected.

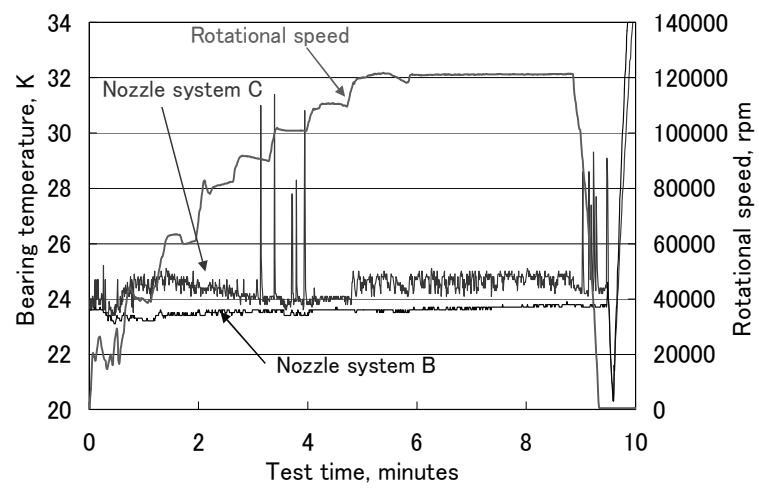

Fig. 10 Change of bearing temperature for bearings used ball B cooled with nozzle systems B and C

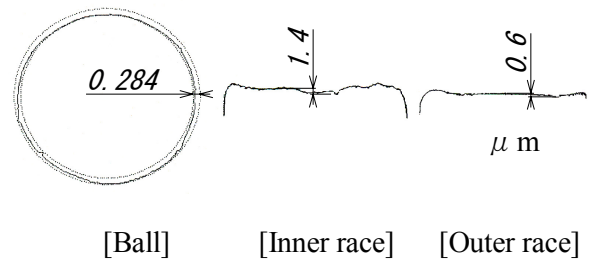

(a) Nozzle system B

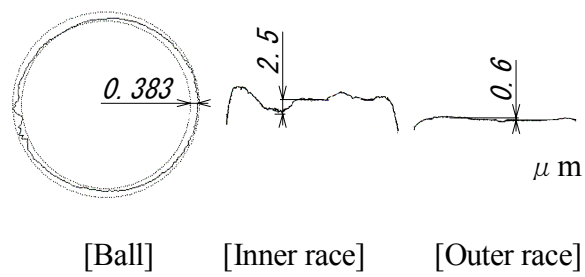

Fig. 11 Wear of ball B and inner-outer raceways cooled with nozzle systems $\mathrm{B}$ and $\mathrm{C}$

For the bearings tested for 30 minutes under thrust loads up to $1,960 \mathrm{~N}$ with nozzle systems $\mathrm{B}$ and $\mathrm{C}$, Fig. 11 shows the deviation from the spherical form of balls and the surface profiles of inner-outer raceways, and Fig. 12 shows typical surface features of the tested ball $B$. The deviation from the spherical form was 0.15-0.28 $\mu \mathrm{m}$ for nozzle system B and $0.18-0.38 \mu \mathrm{m}$ for nozzle system C, respectively. It was noted that, for nozzle system $\mathrm{C}$ accompanied by a significant rise of the bearing temperature, the wear depth of the inner raceway was higher at 1.1-2.5 $\mu \mathrm{m}$. Furthermore, it was clear that microcracks occurred by nozzle system $\mathrm{C}$ were visually severe compared with those for nozzle system B.

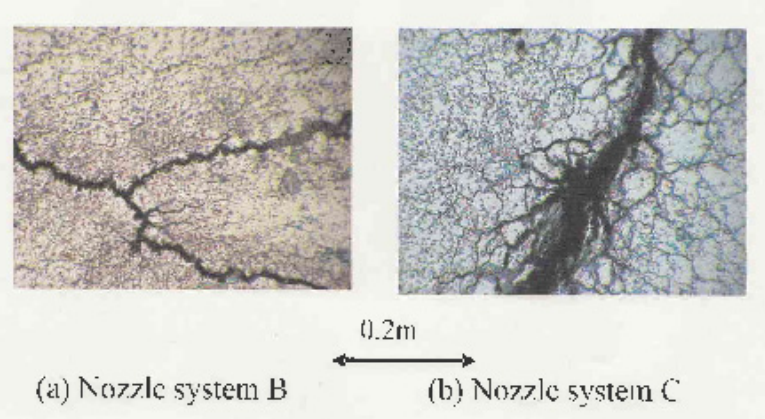

Fig. 12 Surface features of ball B cooled with nozzle systems $\mathrm{B}$ and $\mathrm{C}$

Nozzle system $\mathrm{C}$ distributed half of the cooling flow to the inner-race side by the two nozzles, resulting in a reduction of cooling at the outer-race side. On the other hand, the jet flow collided with the flat side of the retainer and flowed to the outer race through the outerland clearance by centrifugal force.

These differences indicated that microcracks might be generated at the contact track between the ball and the outer raceway under poor cooling conditions due to a narrow outerland clearance. For the jet cooling system, the jet flow aimed at the retainer was superior to that aimed at the inner raceway.

\subsubsection{Numbers of nozzles}

By using all nozzles aimed at the retainer, nozzle system B (with four nozzles) and nozzle system D (with two nozzles) were compared. The jet speed was controlled to be lower, that is, $159 \mathrm{~m} / \mathrm{s}$ for four nozzles and $168 \mathrm{~m} / \mathrm{s}$ for two nozzles, respectively. The bearings with the fragile ball $\mathrm{C}$ due to thermal shock were tested in $\mathrm{LH}_{2}$ for a total time of 30 minutes at speeds to $120,000 \mathrm{rpm}$ under thrust loads to $1,960 \mathrm{~N}$. For both these nozzle types, no remarkable difference of the bearing temperature was detected.

For the bearings cooled with nozzle systems B and D, Fig. 13 shows the deviation from the spherical form of balls and the surface profiles of inner-outer raceways, and Fig. 14 shows typical surface features of the tested ball C. For both tested balls, conditions of wear and microcracks seemed to be severe due to poor thermal shock resistance as compared with those of the tough ball B (as shown in Fig. 11). For four nozzles, microcracks appeared to be narrow compared with those for two nozzles. The wear depth of the inner-outer raceway 
for two nozzles was apt to be higher than that for four nozzles.

To obtain even cooling conditions surrounding the bearing, four nozzles seemed to be better than two nozzles. Furthermore, four nozzles with increasing jet speed to $208 \mathrm{~m} / \mathrm{s}$ could achieve high cooling ability, so that the propagation of microcracks was evidently restrained, as discussed in the previous section.

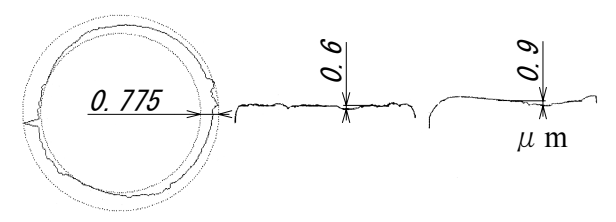

[Ball] [Inner race] [Outer race]

(a) Nozzle system B

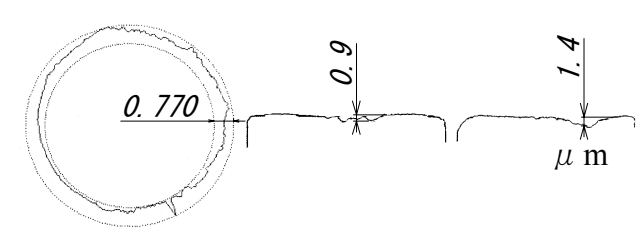

[Ball] [Inner race] [Outer race]

(b) Nozzle system D

Fig. 13 Wear of ball $\mathrm{C}$ and inner-outer raceways cooled with nozzle systems B and D

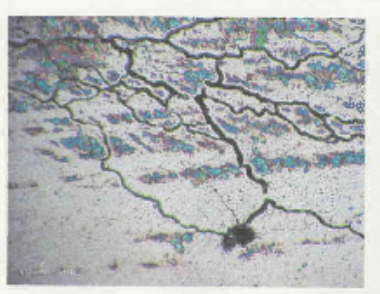

(a) Noz7le system B

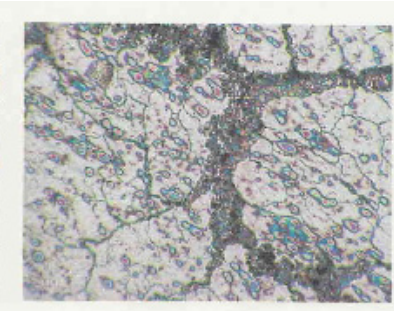

(b) Nozzle system I)
Fig. 14 Surface features of ball C cooled with nozzle systems B and D

\subsubsection{Bearing acceleration}

In an attempt to directly detect the bearing damage such as microcracks that occurred during testing, the BCA $\left(G_{r m s}\right.$ and $\left.G_{p k}\right)$ was monitored. As mentioned above, at the bearing test at a speed of 100,000 rpm under a light thrust load of $980 \mathrm{~N}$, the BCA of the bearings was maintained at a lower level without abnormal sign; however, superficial microcracks were propagated, as shown in Fig. 5. Therefore, with the employed monitoring system of the BCA, it was difficult to detect damage such as superficial microcracks with very shallow depths.
Figure 15 shows the change of the $\mathrm{BCA}$ for the bearings cooled with nozzle systems A, C and D. For the four-nozzle systems $\mathrm{A}$ and $\mathrm{C}$, the bearings used the tough ball B. For the two-nozzle system D, to evaluate the propagation of wide-ditch microcracks, the bearing used the fragile ball A. The bearings were tested in $\mathrm{LH}_{2}$ at speeds up to $120,000 \mathrm{rpm}$ with a thrust load of 1,960 N. Furthermore, Fig. 16 shows the frequency analysis of the BCA displayed three-dimensionally. For nozzle system A with a higher jet-speed $(208 \mathrm{~m} / \mathrm{s})$, it was noted that the BCA conditions were at a relatively lower level, as compared to those for nozzles systems C $(159 \mathrm{~m} / \mathrm{s})$ and $\mathrm{D}(168 \mathrm{~m} / \mathrm{s})$. It was found that $G_{r m s}$ and $G_{p k}$ overlapped and resulted in a normal condition without surface damage of the balls, as compared with those of nozzle systems $\mathrm{C}$ and $\mathrm{D}$.

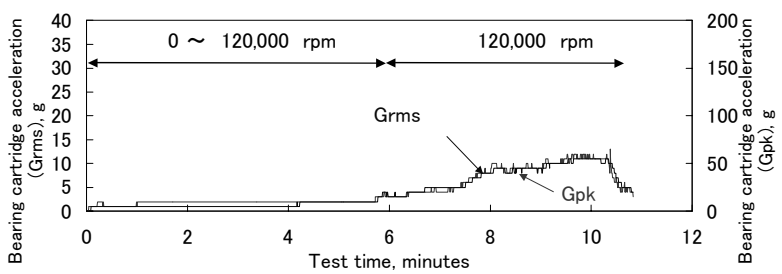

(a) Nozzle systems A (with ball B)

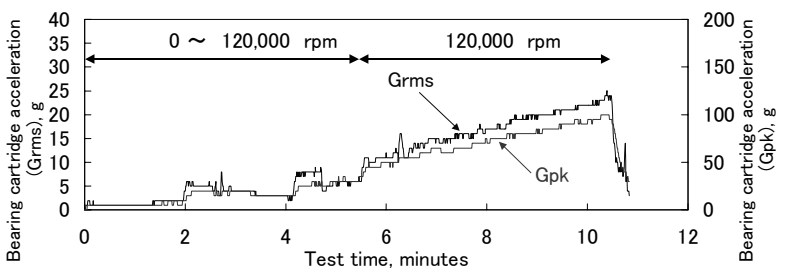

(b) Nozzle systems C (with ball B)

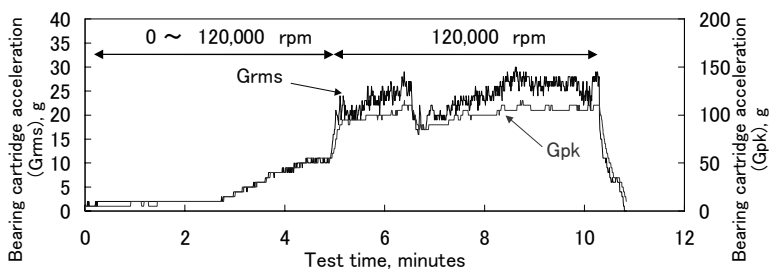

(c) Nozzle systems D (with ball A)

Fig. 15 Change of BCA for bearings cooled with nozzle systems A, C and D

Furthermore, for the nozzle system A, the BCA at a frequency of about $8 \mathrm{kHz}$ defined by frequency analysis, which was relatively high and produced by the rolling balls, might be decreased due to restraining of microcrack propagation. In contrast, under poor cooling conditions for nozzle systems $\mathrm{C}$ and $\mathrm{D}$, the BCA depending on the rolling balls was considerably high due to an increase of the surface damage on the balls. For the bearing having the fragile ball A cooled with system D, the BCA of balls detected a significant increase due to the propagation of wide-ditch cracks. To the contrary, it was shown that, for the bearing used the tough ball B, 
change of the BCA was stable and moderate under nozzle systems $\mathrm{A}$ and $\mathrm{C}$.

Thus, the BCA analysis indicated that the jet cooling through four nozzles aimed at the retainer side achieved higher cooling ability with increasing jet speed above $208 \mathrm{~m} / \mathrm{s}$. Furthermore, it was detected that toughness of the ball B was superior to that of the ball A resulting in the higher propagation of microcracks.

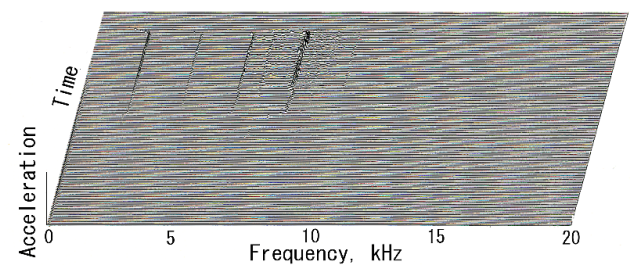

(a) Nozzle systems A (with ball B)

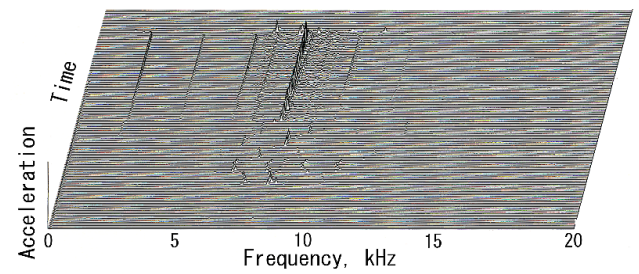

(b) Nozzle systems C (with ball B)

(b)

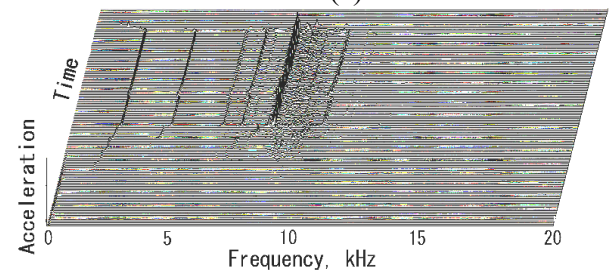

(c) Nozzle systems D (with ball A)

Fig. 16 Frequency analysis of BCA (visualized in threedimensional display)

\subsection{Performance of modified bearings}

\subsubsection{Bearing tests}

Based on the results discussed in the previous sections, superficial microcracks seemed to be formed at the contact track between the ball and the outer raceway under insufficient cooling conditions in addition to a large centrifugal force. Superficial microcracks thus seem to be produced by thermal fracture due to high heat generation, promoted by rupture of the durable $\mathrm{CaF}_{2} / \mathrm{FeF}_{2}$ transfer film ${ }^{7)}$ withstanding high stress. For the modified bearing, to prevent the rupture of transfer film at the outer raceway, the outer-race curvature was minimized to 0.51 and thus the contact stress level was possibly reduced.

The modified bearings A (the outerland clearance of the retainer, $0.2-0.3 \mathrm{~mm})$ and $B(0.1-0.2 \mathrm{~mm})$ were tested for a total time of 26 minutes at speeds to 120,000 rpm and thrust loads to $1,960 \mathrm{~N}$. Furthermore, to clarify the effect of stress reduction at the outer race, these modified bearings used the brittle ball $\mathrm{C}$ which exhibited higher bearing temperature, as shown in Fig. 9. The bearings were effectively cooled with four-nozzle system A with a high jet speed of $208 \mathrm{~m} / \mathrm{s}$.

Figure 17 shows the change of the bearing temperature and the BCA for modified bearings A and B under a thrust load of $1,960 \mathrm{~N}$. Both bearings exhibited a stable variation of the bearing temperature as compared with those of the conventional bearing using ball $\mathrm{C}$. Though the ball $\mathrm{C}$ indicated poor bearingperformance because of lower thermal shock resistance, the erratic change of the bearing temperature could be restrained by decreasing the outer-race stress. For the bearing B with a narrow outerland clearance, $G_{r m s}$ of the BCA during testing at a speed of $120,000 \mathrm{rpm}$ drastically increased and indicated an increase of the surface roughness due to insufficient cooling at the outer race.

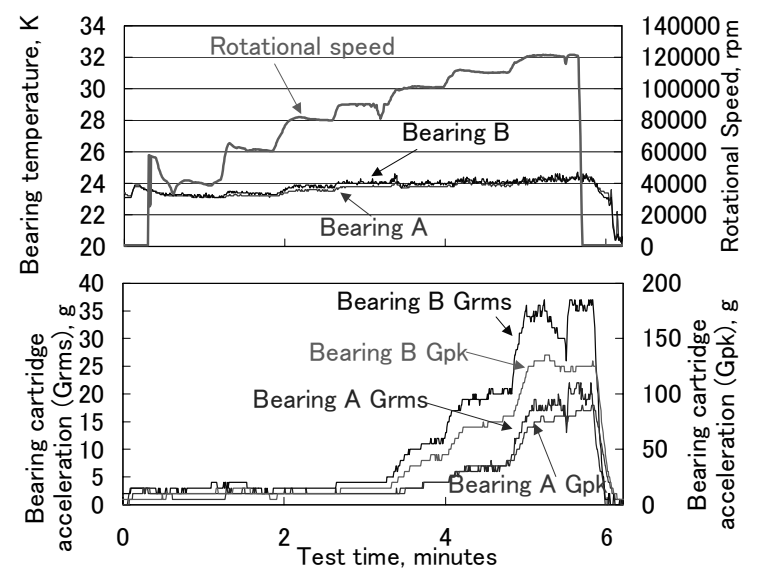

Fig. 17 Change of bearing temperature and $\mathrm{BCA}$ for modified bearings $\mathrm{A}$ and $\mathrm{B}$ used ball $\mathrm{C}$

Figure 18 shows typical surface features of the balls $\mathrm{C}$ used in the modified bearings. For the bearing $\mathrm{A}$ whose outer race was sufficiently cooled, superficial microcracks were hardly observed on any of the balls. In contrast, the bearing B exhibited sporadic spreading of microcracks due to poor cooling of the outer race. The deviation from the spherical form of balls was $0.17-0.26 \mu \mathrm{m}$ for the bearing A (without microcracks) and $0.15-0.41 \mu \mathrm{m}$ for the bearing $\mathrm{B}$ (with slight microcracks). Although the wear depth of inner raceways was 1.1-1.7 $\mu \mathrm{m}$ for both bearings, the wear depth of outer raceway for bearing A was 0.7-1.0 $\mu \mathrm{m}$ and was lower than that of 1.3-1.5 $\mu \mathrm{m}$ for bearing B. Thus, the above results indicated that, at the outer race, the reduction of the contact stress sustaining the higher centrifugal force, as well as the sufficient cooling, were essential to restrain the propagation of thermal microcracks. 

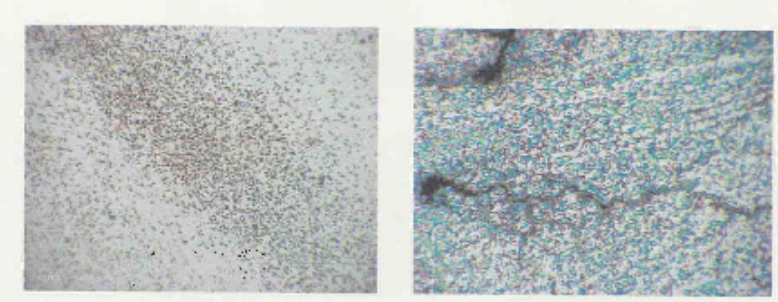

(a) Bearing $A$ †. $2 \mathrm{~mm}$ (b) Betring $B$

Fig. 18 Surface features of ball $\mathrm{C}$ used in modified bearings $\mathrm{A}$ and $\mathrm{B}$

\subsubsection{Critical load capacity at inner/outer raceways}

It was clarified that the modified bearing with a limit outer-race curvature of 0.51 could restrain the propagation of superficial microcracks at a speed of $120,000 \mathrm{rpm}$ and a thrust load of $1,960 \mathrm{~N}$. Table 5 summarizes the contact load, stress and spin velocity conditions calculated at above operating conditions for the modified bearing as compared with those of the conventional bearing. From these calculated results, the critical load capacity (stress) of the transfer film enabling to withstand high contact stress was evaluated.

Table 5 Contact load, stress and spin velocity conditions calculated at 120,000 rpm and thrust load of 1,960 N

\begin{tabular}{|c|c|c|c|}
\hline \multirow{2}{*}{$\begin{array}{c}\text { OUTER RACE } \\
\text { CURVATURE }\end{array}$} & \multicolumn{3}{|c|}{ INNER RACE } \\
\cline { 2 - 4 } & Contact load (N) & Maximum stress (GPa) & $\begin{array}{c}\text { Maximum spin } \\
\text { velocity (m/s) }\end{array}$ \\
\hline 0.52 & 512 & 2.83 & 6.60 \\
\hline 0.51 & 545 & 2.89 & 6.20 \\
\hline
\end{tabular}

\begin{tabular}{|c|c|c|c|}
\hline OUTER RACE & \multicolumn{3}{|c|}{ OUTER RACE } \\
\cline { 2 - 4 } CURVATURE & Contact load (N) & Maximum stress (GPa) & Centrifugal force (N) \\
\hline 0.52 & 921 & 2.30 & 436 \\
\hline 0.51 & 961 & 2.02 & 432 \\
\hline
\end{tabular}

For the modified bearing, the maximum contactstress of the outer race could be reduced to $2.0 \mathrm{GPa}$ (the outer-race contact load in addition to centrifugal force, $961 \mathrm{~N})$ as compared with that of $2.3 \mathrm{GPa}(921 \mathrm{~N})$ for the conventional bearing. On the other hand, at the inner raceway with ball spinning because of the outer-race control under high speeds, the maximum stress was in a level of 2.8-2.9 GPa (the inner-race contact load, 512$545 \mathrm{~N}$ ), and the spin velocity was $6.2-6.6 \mathrm{~m} / \mathrm{s}$. The ball-spin conditions for the modified bearing were almost the same as those for the conventional bearing.

It should be noted that the outer-race contact load increased with addition of centrifugal force and reached a level twice that of the inner-race contact load. Under such severe load conditions, rapture of the transfer film would lead to a significant increase of frictional heating. At the $\mathrm{LH}_{2}$ temperature, the specific heats and the thermal conductivities of the bearing components drop off rapidly and local hot spots are easily produced at the outermost surface in boiling $\mathrm{LH}_{2}$.

As mentioned above, under a maximum contact stress above $2.0 \mathrm{GPa}$ at the insufficiently cooled outer raceway, rupture of the transfer film possibly occurred, so that severe heated spots frictionally occurred on the $\mathrm{Si}_{3} \mathrm{~N}_{4}$ balls at the contact track against the outer race. Abrupt cooling at hot spots in $\mathrm{LH}_{2}$ certainly added high thermal stress to maximum contact stress, resulting in the propagation of superficial microcracks.

The critical outer-race contact stress of $2.0 \mathrm{GPa}$ within the hybrid ceramic bearing was consequently the same as that in a previous study ${ }^{1)}$, which was conducted for the all-steel bearings operating with the local film rupture, as defined by the electrical resistance monitoring in boiling $\mathrm{LH}_{2}$. Thus, to restrain the propagation of microcracks, it is important to decrease the outer-race stress possibly while increasing outer-race cooling through a narrow outerland clearance of the retainer.

On the other hand, sufficient cooling was obtained at the inner race because of the relieved inner ring. By another test, it was found that, at a speed of 120,000 rpm, the critical load capacity at the inner raceway without bearing damage was estimated to be about 3.0 GPa with a spin velocity of $6.7 \mathrm{~m} / \mathrm{s}$ under a thrust load of $2,160 \mathrm{~N}^{7)}$. Furthermore, it was shown that the critical load capacity was in a range of 2.7-2.9 GPa for the seized all-steel bearings in sufficient cooling conditions of $\mathrm{LN}_{2}{ }^{1)}$. Thus, at the inner race cooled sufficiently, the critical contact stress was probably $3.0 \mathrm{GPa}$ to restrain the propagation of thermal microcracks.

\section{Conclusions}

The Durability of the hybrid ceramic 25-mm-bore bearings having a single land guided-retainer was evaluated and improved in $\mathrm{LH}_{2}$ at speeds to 120,000 rpm ( 3 million DN) and thrust loads to $1,960 \mathrm{~N}$. To evaluate durability under insufficient cooling conditions, the cooling flow rate for each bearing was restrained to about 0.32 liters/s. To select a tough $\mathrm{Si}_{3} \mathrm{~N}_{4}$ ball capable of restraining microcrack propagation, bearing tests were conducted for three kinds of the candidate $\mathrm{Si}_{3} \mathrm{~N}_{4}$ balls with different jet-cooling systems. The following results were obtained:

(1) The bearing used the tough $\mathrm{Si}_{3} \mathrm{~N}_{4}$ ball (ball B) having high fracture toughness as well as high thermal shock resistance could restrain the propa- gation of superficial wide-ditch microcracks on the balls. Such wide-ditch cracks seemed to be formed by removal of fragments fractured along hair cracks.

(2) The cooling ability of the jet flow aimed at the retainer was superior to that aimed at the inner 
raceway because sufficient supply of the cooling flow through a narrow outerland clearance was conducted to the outer raceway withstanding a large centrifugal force.

(3) The jet cooling through four nozzles aimed at the retainer side was able to achieve higher cooling ability with increasing jet speed above $208 \mathrm{~m} / \mathrm{s}$, the jet speed of which reached a level twice that of the sliding speed at the outerland of the retainer.

(4) Microcracks on the balls were probably generated due to rupture of the transfer film at the trace contacting against the outer raceway under severe contact stress (above $2.0 \mathrm{GPa}$ ) increased by high centrifugal load accompanied by insufficient cool- ing conditions.

(5) Decreasing the outer-race stress to $2.0 \mathrm{GPa}$ by a limited race curvature of 0.51 in conjunction with sufficient cooling through a narrow outerland clearance prevented the propagation of superficial thermal microcracks.

\section{Acknowledgments}

This paper was based on a previous study carried out by Japan Aerospace Exploration Agency (JAXA) at Kakuda Space Center. This work was supported by NTN corporation, which supplied the test bearings, inspected the tested bearings, conducted thermal-shock and crush-load tests as well as the bearing analysis. The authors are indebted to T. Itou of NTN and workers engaged for their valuable support and to those organizations for their enthusiastic cooperation.

\section{References}

[1] Nosaka, M., Oike, M., Kikuchi, M., Kamijo, K. and Tajiri, M., "Tribo-Characteristics of Self-Lubricating Ball Bearings for the LE-7 Liquid Hydrogen Rocket-Turbopumps," Trib. Trans., 36, 3, 1993, 432-442.

[2] Wang, L., Snidle, R. W. and Gu, L., "Rolling Contact Silicon Nitride Bearing Technology: A Review of Recent Research," Wear, 246, 2000, 159-173.

[3] Ohta, T., Kimoto, K., Kawai, T., Motomura, T., Russ, M. and Paulus, T., "Design, Fabrication and Test of the RL60 Fuel Turbopump," Preprint No. AIAA-2003-5073, 2003.
[4] Bosson, R., Edeline, E. and Collongeat, L., "High Performance Cryogenic Ball Bearing Demonstration," Preprint No. AIAA-99-2346, 1996.

[5] Bursey, R. W. Jr., Chin, H. A., Oliger, J. B., Moore, L. C., Thom, R. L., Moore, J. D. and Marty, D. E., "Advanced Hybrid Rolling Element Bearings for the Space Shuttle Main Engine High Pressure Alternate Turbopumps," Preprint No. AIAA-96-3101, 1996.

[6] Nosaka, M., Oike, M., Kikuchi, M. and Mayumi, T., "Tribo-Characteristics of Cryogenic Hybrid Ceramic Ball Bearings for Rocket Turbopumps: Self-Lubricating Performance," Trib. Trans., 40, 1, 1997, 21-30.

[7] Nosaka, M., Kikuchi, M., Oike, M. and Kawai, N., "Tribo-Characteristics of Cryogenic Hybrid Ceramic Ball Bearings for Rocket Turbopumps: Bearing Wear and Transfer Film," Trib. Trans., 42, 1, 1999, 106-115.

[8] Nosaka, M., Takada, S., Kikuchi, M., Sudo, T. and Yoshida, M., "Ultra-High-Speed Performance of Ball Bearings and Annular Seals in Liquid Hydrogen at Up to 3 Million DN (120,000 rpm)," Trib. Trans., 36, 3, 2004, 43-53.

[9] Collongeat, L., Edeline, E., Frocot, M. and Dehouve, J., "Development Status of High DN $\mathrm{LH}_{2}$ Bearings in Snecma," Preprint No. AIAA-2005-3950, 2005.

[10] Gu, L., Wang, L., Jin, Y. and Li, X., "Fatigue Behavior of Hybrid Ceramic Ball Bearings in Liquid Nitrogen," Trib. Trans., 46, 4, 2003, 494-498.

[11] Rachuk, V. and Titkov, N., "The First Russian LOX-LH $H_{2}$ Expander Cycle LRV: RD0146," Preprint No. AIAA-2006-4904, 2006.

[12] Nosaka, M., Takada, S., Kikuchi, M., Sudo, T. and Yoshida, M., "Evaluation of Durability of Hybrid Ceramic Ball Bearings in Liquid Hydrogen at 3 Million DN (120,000 rpm)," Preprint No. ISTS 2006-a-17, 2006.

[13] Aksel, C. and Warren, P. D., "Thermal Shock Parameters [R, R" and R"''] of Magnesia-Spinel Composites," J. of the European Ceramic Society, 23, 2003, 301-308.

[14] Nosaka, M., Oike, M., Kikuchi, M., Kamijo, K. and Tajiri, M., "Self-Lubricating Performance and Durability of Ball Bearings for the LE-7 Liquid Oxygen Rocket-Turbopump," Lubr. Eng., 49, 9, 1993, 677-688. 